Trauma Surgery \& Acute Care Open

\title{
Direct admission to improve timely access to care for patients requiring transfer to a level 1 trauma center
}

\author{
Kyan C Safavi, ${ }^{1,2}$ Apostolos Gaitanidis, 1,3 Kerry Breen, 1,3 Mark Seelen, ${ }^{1,3}$ Ali Raja, ${ }^{1,4}$ \\ George C Velmahos, ${ }^{1,3}$ Peter F Dunn ${ }^{1,2}$
}

\begin{abstract}
'Massachusetts General Hospital, Boston, Massachusetts, USA

${ }^{2}$ Anesthesia, Critical Care and Pain Medicine, Massachusetts General Hospital, Boston, MA, USA

${ }^{3}$ Surgery, Massachusetts General Hospital, Boston, MA, USA ${ }^{4}$ Emergency Medicine, Massachusetts General Hospital, Boston, MA, USA
\end{abstract}

\section{Correspondence to} Dr Kyan C Safavi, Massachusetts General Hospital, Boston, MA 02114-2696, USA; ksafavi@ mgh.harvard.edu

New England Surgical Society, Newport, Rhode Island, USA, 2020.

Received 22 September 2020 Revised 19 November 2020 Accepted 30 November 2020

(C) Author(s) (or their employer(s)) 2020. Re-use permitted under CC BY-NC. No commercial re-use. See rights and permissions. Published by BMJ.

To cite: Safavi $K C$ Gaitanidis A, Breen K, et al. Trauma Surg Acute Care Open 2020;5:e000607.

\section{ABSTRACT}

Background Emergency departments (EDs) at level 1 trauma centers are often overcrowded and deny ED-toED transfers from lower-tiered centers. Lack of access to timely level 1 care is associated with increased mortality. We evaluated the feasibility of a direct admission (DA) protocol as a method to increase timely access to a level 1 trauma center during periods of ED overcrowding. Methods During periods of ED overcrowding between 1 May and 31 December 2019, we admitted patients from referring EDs directly to the intensive care unit (ICU) or inpatient ward using the DA protocol. In a prospective comparative study design, we compared their outcomes to patients during the same period who were admitted through the ED when the ED was not overcrowded. Results During periods of ED overcrowding, transfer was requested and clinically accepted for 28 patients, of which 23 (82.1\%, age $63 \pm 20.3$ years, men $52.2 \%$ men) were successfully admitted via the DA protocol. Five $(17.9 \%)$ were not successfully transferred due to lack of available inpatient beds. During periods when the ED was not overcrowded, 106 patients (age 62.8 23.1 years, men $52.8 \%$ ) were admitted via the ED. There were no morbidity or mortality events attributed to the DA process. Time to patient arrival was 2.7 hours $(95 \% \mathrm{Cl}$ 2.3 to 3.1$)$ in the DA cohort and 1.9 hours $(95 \% \mathrm{Cl} 1.5$ to 2.4 ) in the ED-to-ED cohort ( $p=0.104)$. Up-triage to the ICU within 24 hours was performed in only one patient (4.3\%). In-hospital mortality did not differ (3 (13\%) vs. 8 (7.6\%), $p=0.392$ ).

Discussion The DA pathway is a feasible method to safely transfer patients from a referring ED to a highercare trauma center when its ED is overcrowded. Level of evidence Level III, care management.

\section{INTRODUCTION}

Access to care at high-level trauma centers for severely injured patients is critical to achieving optimal outcomes. ${ }^{12}$ In an effective trauma system, severely injured patients who are taken to a lowerlevel or non-trauma center are first stabilized and then promptly transferred to higher-level care. ${ }^{3}$

The transfer process, however, is under threat from increasingly congested emergency departments (EDs). ED overcrowding can lead to delays in transfer and worse patient outcomes. ${ }^{4-6}$ Policy makers have taken notice of the challenges presented by congested EDs. ${ }^{78}$ The Commonwealth of Massachusetts became the first state to mandate hospital policies that address ED overcrowding. ${ }^{9}{ }^{10}$ Massachusetts now requires all acute care hospitals to implement a 'Code Help' policy when their EDs are unable to accept new patients into licensed treatment spaces. These policies include deferring transfers from referring EDs to prevent suboptimal care in overcrowded areas with inadequate personnel coverage. ${ }^{11}$ Though these policies may reduce ED congestion, they risk exacerbating delays in access to high-level emergency care.

A frequent paradox is that, despite ED congestion, appropriate inpatient beds may be readily available. For example, the ED may be overcrowded with general medicine patients, whereas inpatient trauma or surgical specialized beds are open and available. Therefore, an opportunity exists for trauma patients to bypass the ED and to be admitted directly to the appropriate inpatient location. Direct admission (DA) is an alternative admission pathway in which a patient from a referring ED gains direct access to an inpatient location-either general care, intensive care unit (ICU) or OR-at the receiving hospital and bypasses its ED entirely. DA may be a useful mechanism to ensure timely transfer of severely injured patients from a referring ED to a higher level of care within the trauma system while avoiding further ED congestion.

Little is known about the design of an effective DA protocol or its feasibility. A multidisciplinary approach to DA includes coordination and correct routing of transfer calls, appropriate triage and admission determinations, accurate assignment to levels of care and locations, efficient mobilization and turnover of inpatient beds to accommodate urgent patients, and clear processes for rapid evaluation on arrival by the receiving clinical team.

We report the design and components of an institutional standard protocol for DA at a level 1 trauma center. Our objective was to evaluate the timely transfer of patients and potential adverse events related to the DA process. Our hypothesis was that DA could be effective and safe in enabling transfers.

\section{METHODS}

\section{Study setting and Code Help}

The study was conducted at a quaternary care, level 1 trauma center with 1034 beds located in Boston, Massachusetts. Our hospital is a regional trauma referral center serving the six New England states. Approximately $40 \%$ of trauma patients treated at our hospital are transferred from other hospitals.

Beginning in 2010, to address ED crowding, the Commonwealth of Massachusetts required that all acute care hospitals licensed by the Department of Public Health have a Code Help policy in place. 
Guidance to hospitals from the Department of Public Health states that Code Help status should be initiated when the ED is unable to accept new patients into a licensed treatment space due to high occupancy. In compliance with this policy, our hospital denied transfers from other hospitals' EDs when in Code Help status. Code Help was activated 193 times from January 2016 to December 2018 for a total of 2741 hours. As a result, the transfer of numerous trauma patients was denied. We therefore developed a DA protocol for patients in need of advanced trauma care that would bypass the ED during Code Help by transferring them directly to an appropriate inpatient location.

\section{DA protocol}

The DA protocol was developed by a multidisciplinary team that included physician, nurse and administrative leaders from emergency medicine, trauma emergency operation, surgical critical care, radiology and inpatient bed management. The team was also tasked with developing processes to ensure that the pathway was implemented safely. The final policy and safety processes were implemented on May 1, 2019.

Figure 1 illustrates the DA protocol for trauma patients. If a bed could not be identified within 10 minutes of the request, the transfer was declined so as to give the referring hospital a timely decision so that they could seek transfer to another trauma center. Table 1 lists safety measures implemented in the protocol to ensure the safe transfer of patients.

\section{Study design and population}

We conducted a prospective cohort study of patients exposed to the DA protocol during Code Help and compared them to a contemporaneous control group of patients who were transferred during non-Code Help hours from a referring ED to our

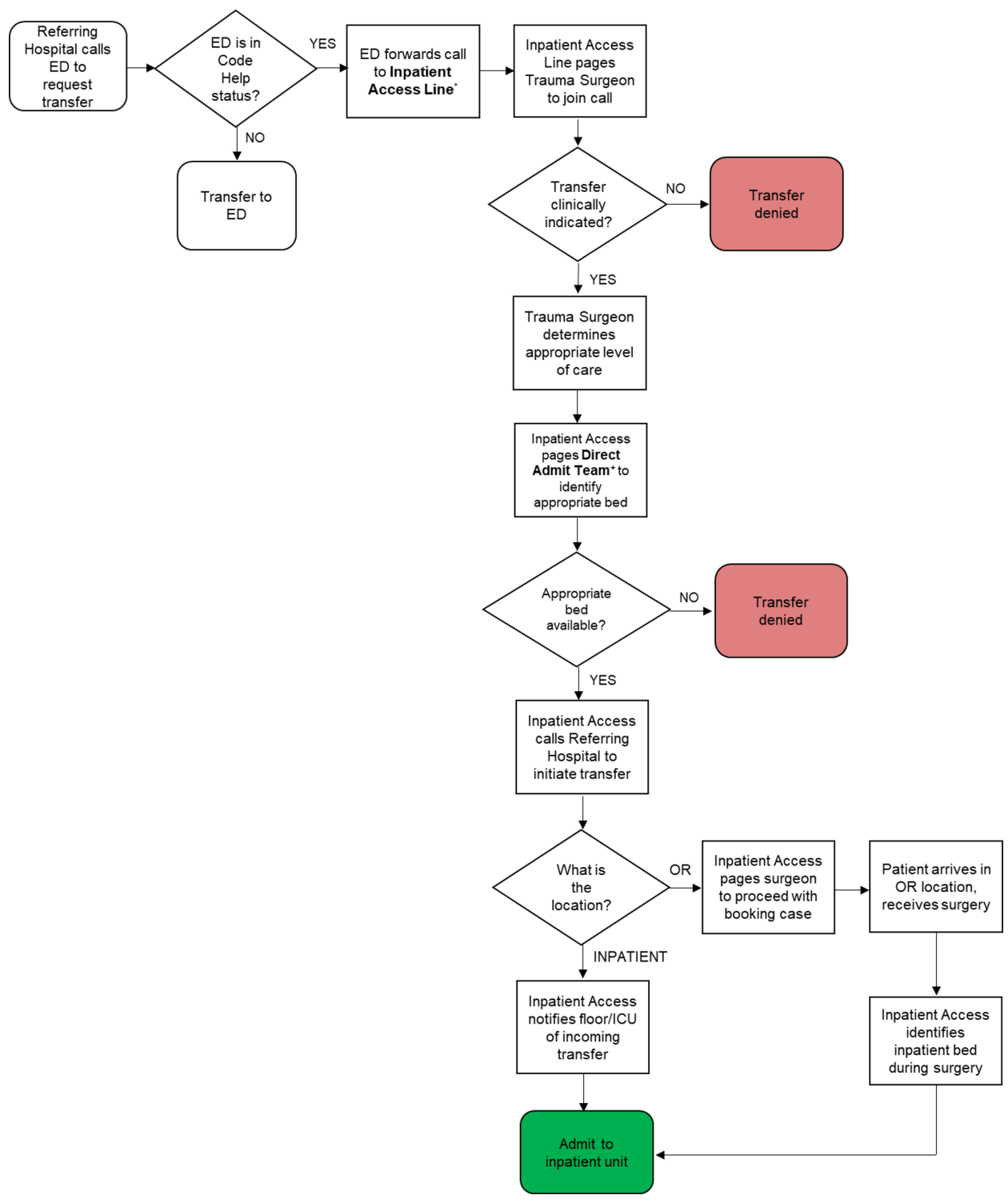

Figure 1 Direct admission pathway for trauma patients. Each step of the pathway is indicated in a box, and steps in which a decision must be made are indicated in the diamonds. *The inpatient access line is a multiway call system staffed 24 hours/day, 7 days/week, by a bed manager. A bed manger is a non-clinician whose scope of practice is assigning inpatient bed locations for new admissions. +The direct admit team is a team of three individuals composed of nursing and physician leadership with knowledge of bed capacity and demand in all inpatient locations and the ED. The team works with the bed manager to discuss options for where a direct admit patient can be admitted. They have the authority to hold existing patients in their current location (eg, PACU) or request transfer of existing patients to a different inpatient location to facilitate a direct. ED, emergency department; ICU, intensive care unit; OR, operating room. 
Table 1 Safety measures for direct admission pathway

\begin{tabular}{|c|c|c|}
\hline Safety measure & Description & Purpose \\
\hline Physician handoff & $\begin{array}{l}\text { Attending surgeon must receive warm handoff from referring hospital prior to } \\
\text { transfer }\end{array}$ & Surgeon determines the appropriate level of intensity of care. \\
\hline Nurse handoff & Nurse must receive warm handoff from referring hospital prior to transfer. & Nurses prepare appropriate personnel and equipment for patient arrival. \\
\hline Team huddle & Nurse and attending surgeon have the option to page for a team huddle. & Team members voice and address any concerns or questions about plan of care. \\
\hline Safe arrival & Team must be at bedside on patient arrival. & Team is present at the bedside when the patient arrives for timely patient assessment. \\
\hline Rapid imaging & If necessary, the team calls a radiology access line to obtain immediate imaging. & Team obtains urgent imaging on arrival. \\
\hline Rapid retriage & $\begin{array}{l}\text { If there is a concern that a patient on arrival requires a higher level of care, trauma } \\
\text { team activates a rapid response to bring additional resources and clinicians to } \\
\text { bedside. }\end{array}$ & $\begin{array}{l}\text { Team can rapidly transfer the patient to an ICU and receive additional resources for the } \\
\text { administration of critical care prior to transfer. }\end{array}$ \\
\hline
\end{tabular}

ED and were thus not exposed to the DA protocol. We included patients from May 1, 2019, to December 31, 2019. Patients were eligible for DA if (1) they were older than 18 years of age; (2) a referring hospital ED requested transfer to our hospital's ED; (3) the referring ED stated that the patient's primary diagnosis was trauma-related (excluding isolated orthopedic extremity fracture $(\mathrm{Fx})$ as hospitals in our trauma network did not advocate for the need to transfer this population urgently); and (4) our hospital's ED was in Code Help status. If patients during the study period met these criteria but our hospital ED was not in Code Help status, the DA protocol was not used and they were transferred in the typical fashion from the referring hospital's ED to our hospital's ED.

\section{Clinical data and outcomes measures}

The DA protocol was implemented as a part of a hospital quality improvement initiative and was exempt from the institutional review. Participants did not provide informed consent as the DA protocol was a quality improvement initiative at the study hospital.

Patients exposed to the protocol were tracked prospectively during their hospitalization by a member of the study team. Data collected included patient age, sex, race, ethnicity, mechanism of injury, hospital length of stay (LOS), ICU LOS and admission Glasgow Coma Scale (GCS). Injury details were classified according to the Abbreviated Injury Scale (AIS) system per the National Trauma Data Bank definitions. The trauma diagnosis known at triage was analyzed by the surgical team in the history and physical admission note. The final trauma diagnosis was analyzed as specified by the surgical team in the patient's discharge summary notes, imaging reports and operative notes.

We measured the incidence of successful transfer for the patients exposed to the DA protocol and the control (ED-to-ED) cohort. The incidence of successful transfer was defined as the number of patients who were physically transferred to our hospital divided by those who were clinically accepted for transfer.

Outcome measures were defined a priori and tracked during the course of the patient's hospital admission. The primary outcome measure was the mortality and morbidity related to the DA process. A group of evaluators composed of trauma surgeons and nurses met during the study period at a weekly peer-reviewed morbidity and mortality conference to examine all complications of patients who were transferred via the DA process. The group analyzed whether the complication was related to the DA protocol. Cases were categorized according to preventability and opportunities for improvement were identified, followed by specific action plans. DA patients without a complication but with an event which risked safety or violated established protocols or processes were reported through an internal 'safety report' mechanism to the corresponding quality assessment officer. After a root cause analysis by the providers involved in the care and other appropriate parties, an action plan was determined to prevent any similar occurrence in the future.

Secondary outcome measures included the rate of adverse events potentially related to transfer among patients transferred using the DA protocol, overall hospital mortality and the timing of CT at the receiving hospital. Adverse events potentially related to transfer were (1) activation of a rapid response or cardiac arrest for a patient triaged to the surgical ward within 24 hours of admission and (2) up-triage of the patient to a higher level of care (ICU) within 24 hours of admission. Timing of CT was analyzed only for patients undergoing CT within 6 hours of admission.

For comparisons between the DA cohort and the control (ED-to-ED) cohort, we used $\chi^{2}$ and Fisher's exact tests to assess statistical differences for categorical values and Student t-test for continuous values. A p value of less than 0.05 was considered statistically significant in all cases. Statistical analysis was performed using STATA V.15.1.

\section{RESULTS}

There were 23 patients admitted via DA during the study period, and 106 patients were admitted via the standard ED-to-ED pathway. Table 2 demonstrates patient characteristics and trauma presentation of the two cohorts. Patients were not different in terms of age, race, ethnicity, ICU LOS or hospital LOS. Overall, trauma mechanism, ISS score, GCS score and most AIS score were not significantly different. A high chest AIS score (3 to 6) and a low external AIS score ( 1 to 2 ) were more frequent in the DA cohort.

The incidence of successful transfer from the referring hospital was $82.1 \%$. Five patients could not be transferred despite activation of the DA protocol. In all cases, the reason that the patient could not be successfully transferred was that a bed could not be identified within 10 minutes, which was the a priori defined turnaround time to decision. Figure 2 shows the location and success of transfers from referring EDs of a lower-level trauma center.

There were no morbidity and mortality events attributed to the DA transfer process. Table 3 illustrates the comparison between the DA and ED-to-ED cohorts. The DA cohort had a lower rate of transfer compared with the ED-to-ED cohort (23, $82.1 \%$ vs. $106,100 \%$; p value $<0.001)$. Time from request to arrival, up-triage to an ICU within 24 hours of arrival and rapid response/cardiopulmonary arrest within 24 hours of arrival, overall hospital mortality, and time to CT scan for those ordered within 6 hours of arrival were not different among the two cohorts. Among the three mortality events in patients in the DA cohort, all were attributable to patient and/or family wishes to 


\begin{tabular}{|c|c|c|c|}
\hline & DA $(n=23)$ & ED to ED $(n=106)$ & $P$ value \\
\hline Age (years) & $63 \pm 20.3$ & $62.8 \pm 23.1$ & 0.962 \\
\hline Sex, male n (\%) & $12(52.2)$ & $56(52.8)$ & 0.954 \\
\hline Race, n (\%) & & & 0.713 \\
\hline American Indian & - & $1(0.9)$ & \\
\hline Asian & - & $3(2.8)$ & \\
\hline Black or African & $1(4.4)$ & $1(0.9)$ & \\
\hline Other race & $1(4.4)$ & $7(6.6)$ & \\
\hline Unknown & - & $2(1.9)$ & \\
\hline White & $21(91.3)$ & $92(86.8)$ & \\
\hline Ethnicity, n (\%) & & & 0.732 \\
\hline Hispanic or Latino & $1(4.4)$ & $7(6.6)$ & \\
\hline $\begin{array}{l}\text { Non-Hispanic or } \\
\text { Latino }\end{array}$ & $22(95.7)$ & $97(91.5)$ & \\
\hline Unknown & - & $2(1.9)$ & \\
\hline $\begin{array}{l}\text { Mechanism of injury, } \\
\text { n (\%) }\end{array}$ & & & 0.231 \\
\hline Blunt & $22(95.7)$ & $105(99.1)$ & \\
\hline Assault & - & $3(2.9)$ & \\
\hline Drowning & - & 1 (1) & \\
\hline Fall & $13(59.1)$ & $74(70.5)$ & \\
\hline MVC & $5(22.7)$ & $22(21)$ & \\
\hline Motorcycle & $1(4.6)$ & $4(3.8)$ & \\
\hline Struck by object & $1(4.6)$ & $1(1)$ & \\
\hline Other & $2(9.1 \%)$ & - & \\
\hline Penetrating & $1(4.4 \%)$ & $1(0.9 \%)$ & \\
\hline GCS score, n (\%) & & & 0.218 \\
\hline $3-8$ & $4(17.4)$ & $7(6.6)$ & \\
\hline $9-12$ & $1(4.4)$ & $3(2.8)$ & \\
\hline $13-15$ & $18(78.3)$ & $96(90.6)$ & \\
\hline Admission unit, $\mathrm{n}(\%)$ & & & 0.611 \\
\hline ICU & $12(52.2)$ & $50(47.2)$ & \\
\hline Floor & $9(39.1)$ & $54(50.9)$ & \\
\hline Operating room & - & $2(1.9)$ & \\
\hline Hospital LOS, mean days & $9 \pm 16.3$ & $6.2 \pm 6.4$ & 0.337 \\
\hline ICU LOS, mean days & $10.5 \pm 13.9$ & $5.3 \pm 6.7$ & 0.403 \\
\hline ISS score, mean & $13.1 \pm 11.8$ & $14.8 \pm 8.1$ & 0.400 \\
\hline Head AIS score, n (\%) & & & 0.536 \\
\hline $1-2$ & $8(34.8 \%)$ & $25(23.6 \%)$ & \\
\hline $3-6$ & $4(17.4 \%)$ & $21(19.8 \%)$ & \\
\hline Face AIS score & & & 0.149 \\
\hline $1-2$ & $8(34.8)$ & $22(20.8)$ & \\
\hline $3-6$ & - & - & \\
\hline Chest AIS score, n (\%) & & & 0.041 \\
\hline $1-2$ & $10(43.5)$ & $65(61.3)$ & \\
\hline $3-6$ & $2(8.7)$ & $1(0.9)$ & \\
\hline $\begin{array}{l}\text { Abdomen AIS score, } \\
\text { n (\%) }\end{array}$ & & & 0.071 \\
\hline $1-2$ & $1(4.4)$ & $26(24.5)$ & \\
\hline $3-6$ & - & $2(1.9)$ & \\
\hline $\begin{array}{l}\text { Extremity AIS score, } \\
\mathrm{n}(\%)\end{array}$ & & & 0.331 \\
\hline $1-2$ & $6(26.1)$ & $44(41.5)$ & \\
\hline
\end{tabular}

\begin{tabular}{|c|c|c|c|}
\hline & $\mathrm{DA}(\mathrm{n}=23)$ & ED to $E D(n=106)$ & $P$ value \\
\hline $3-6$ & - & $1(0.9)$ & \\
\hline External AIS score, n (\%) & & & 0.002 \\
\hline $1-2$ & $19(82.6)$ & $50(47.2)$ & \\
\hline
\end{tabular}

AIS, Abbreviated Injury Scale; DA, direct admission; ED, emergency department; GCS, Glasgow Coma Scale; ICU, intensive care unit; ISS, Injury Severity Scale; LOS, length of stay; MVC, motor vehicle crash.

focus on end-of-life care in the setting of severe injuries and were not related to the DA process.

There were $6(26.1 \%)$ patients in the DA cohort who received an operating room procedure after transfer. These included replacement of the ascending aorta, rib Fx fixation, hemicraniectomy, cervical spine fusion and open reduction of a femur Fx, and stenting of the subclavian artery.

\section{DISCUSSION}

To address challenges with ED crowding in our level 1 trauma center that result in delays or denials of transfers, we designed and implemented a protocol to increase timely access to care. In a prospective cohort study, we found that DA was effective more than $80 \%$ of the time in enabling access to high-level trauma care at our institution. Previous to implementation of the DA protocol, these patients would have been denied access. In addition, DA appears to be safe. There were no morbidity or mortality events attributed to the DA process after quality review by a group of evaluators composed of trauma surgeons and nurses. In addition, adverse events such as up-triage to an ICU, rapid response and cardiopulmonary arrest within 24 hours of transfer using DA were no different from transfers via the ED during the same period. Overall hospital mortality was also not different between the two cohorts.

Our findings offer evidence in support of DA as a viable strategy for level 1 or 2 trauma centers with highly congested EDs that threaten the timely transfer of trauma patients. Though we found a trend toward transfers in the DA cohort taking longer to arrive at our hospital by approximately $45 \mathrm{~min}$, this difference was not statistically significant. We found that all transfers in the DA cohort who were successfully transferred had a time from initial call to decision communicated to the referring ED within the a priori defined target of 10 minutes. It is unclear whether there may be other aspects of the DA process that may cause a delay.

Though we cannot conclude that without the DA process patients would have received inadequate trauma care, we expect that the DA process is likely efficacious in ensuring more timely care for the patient, given that the selection of which level 1 trauma center to transfer a patient is typically (though not always) based on geographical proximity to the referring hospital. Thus, the DA process increases the likelihood that the patient gets access to the geographically more proximate hospital without needing to call additional hospitals for transfer that are less likely to be geographically as close by.

To our knowledge, our study is the first to report on the design and feasibility of DA for trauma patients. Previous literature regarding the development of strategies to ensure timely transfer to high-level care has focused on the geographical distribution of facilities and their proximity to patients. ${ }^{12-14}$ Several studies have demonstrated that delays in transfer to a higherlevel trauma center result in worse outcomes. ${ }^{1-3}$ The American College of Surgeons recently published a study concluding that 


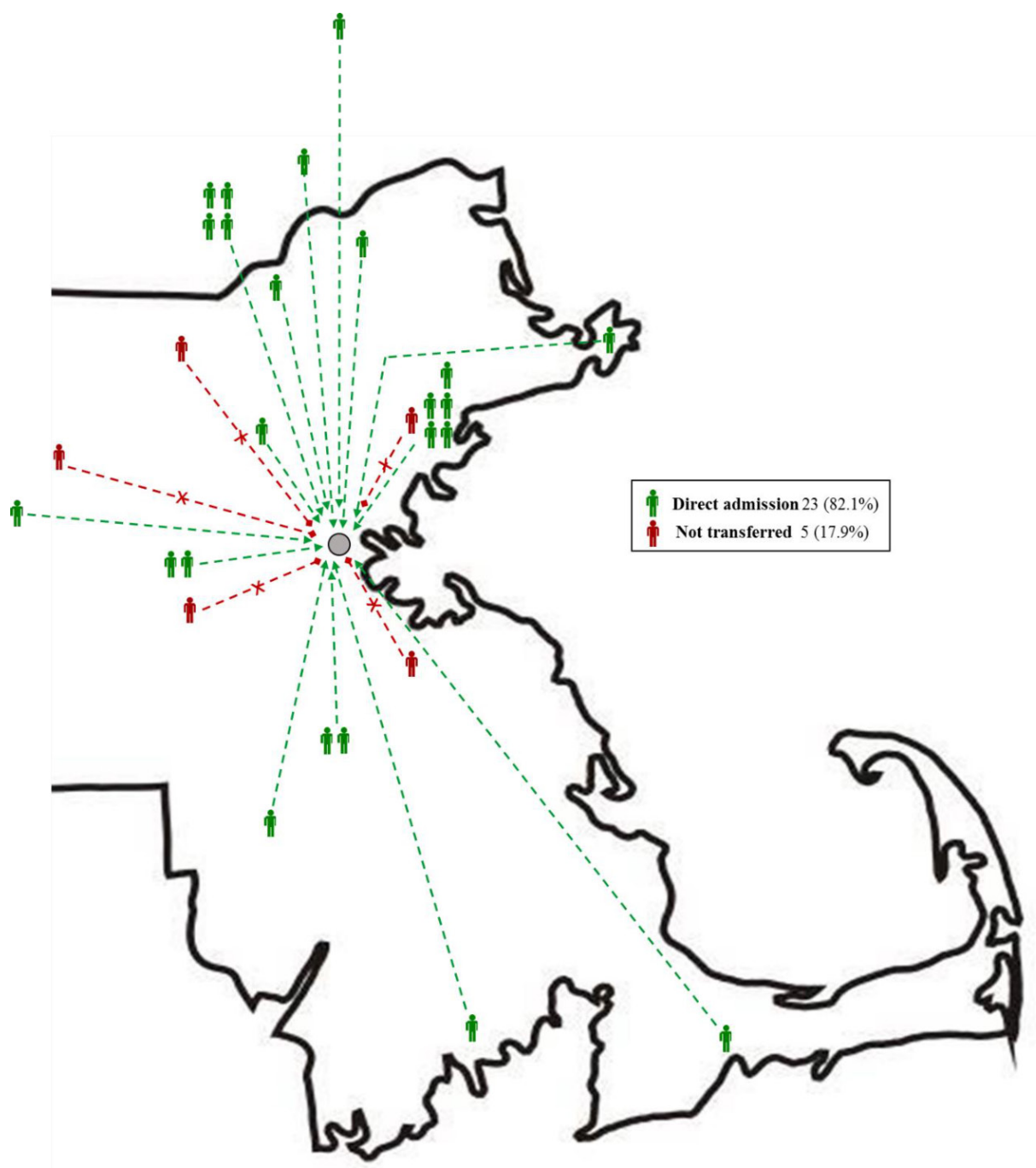

Figure 2 Emergency department locations of lower-level trauma centers for direct admission attempts which were transferred versus not transferred. Patients who were successfully transferred are indicated in green. Patients who were not transferred are indicated in red.

'access delayed is accessed denied'. ${ }^{15}$ In their study, Hashmi et al demonstrated that among more than 1.9 million trauma patients, states with better access to trauma care had lower age-adjusted mortality. DA represents one strategy to help ensure the optimization of patient safety in trauma system care and potentially to improve outcomes. Future studies should explore this relationship.

Several aspects of the DA protocol were essential to ensure appropriate care for these patients, and hospitals designing DA protocols should be attentive to these. First, the presence of the trauma team on arrival of the patient in the general care ward or ICU was critical. In many trauma bays at high-level centers, trauma team members maintain a continuous presence within the ED and are therefore present to ensure rapid assessment of the patient on arrival. In DA, patients are transferred directly to the inpatient location, which may not have trauma team members located there continuously. Within our DA protocol, when the trauma surgeon accepted the patient for transfer, trauma team members were informed about the expected arrival time of the patient, and a member of the trauma team, the bedside nurse and the nurse manager were designated to be present on the arrival of the patient. Primary surveys were performed for all patients at the time of arrival by the trauma team at the bedside, which included an attending and resident physician, as well as two or more nurses (bedside nurse and resource nurse).

In addition, the trauma team at the bedside that received the patient analyzed whether any further imaging was needed. For any imaging studies that were emergently indicated, the trauma team followed a protocol using an order system that indicated urgency and a hotline to connect with the radiologist. Radiologists at our hospital were educated about DA and were prepared to prioritize the patient for rapid imaging when indicated by the operation team. Ensuring ready access to imaging may be a challenge for hospitals. Hospitals implementing DA protocols should consider tracking metrics such as time to CT scan. In our study, this metric was similar for DA and ED-to-ED cohorts.

Given the impact of ED crowding on patient safety, an important future direction of DA would be to analyze whether such a pathway could be implemented to prevent ED overcrowding rather than purely in reaction to it, as our pathway was designed. By routinely performing DA in trauma patients, the ED may be less crowded with acutely injured patients and may be better able to care for those entering the ED from the street.

This study has several limitations. First, it was designed and implemented in a single center. The feasibility and effectiveness of the DA protocol at our institution may not be generalizable to 
Table 3 Outcomes of direct admission and control cohorts

\begin{tabular}{|c|c|c|c|}
\hline & $\begin{array}{l}\text { Direct admission } \\
(\mathrm{n}=23)\end{array}$ & ED to $E D(n=106)$ & P value \\
\hline Transferred, n (\%) & & & $<0.001$ \\
\hline Yes & $23(82.1)$ & $106(100)$ & \\
\hline No & $5(17.9)$ & $0(0)$ & \\
\hline Time to arrival, hours $(95 \% \mathrm{Cl})$ & 2.7 (2.3 to 3.1$)$ & 1.9 (1.5 to 2.4$)$ & 0.104 \\
\hline Up-triage within 24 hours, n (\%) & & & 0.178 \\
\hline Yes & $1(4.3)$ & - & \\
\hline No & $22(95.7)$ & $106(100)$ & \\
\hline $\begin{array}{l}\text { Rapid response/cardiopulmonary arrest } \\
\text { within } 24 \text { hours of admission, } n(\%)\end{array}$ & & & 1.000 \\
\hline Yes & - & $2(1.8)$ & \\
\hline No & $23(100)$ & $104(98.2)$ & \\
\hline Mortality, n (\%) & $3(13.0)$ & $8(7.6)$ & 0.392 \\
\hline $\begin{array}{l}\text { Morbidity/mortality events attributed to } \\
\text { direct admit process, } \mathrm{n}(\%)\end{array}$ & $0(0)$ & - & - \\
\hline Discharge disposition, $\mathrm{n}(\%)$ & & & 0.670 \\
\hline Acute care facility & - & $3(2.8)$ & \\
\hline Correctional facility & - & $1(0.9)$ & \\
\hline Home or self-care & $9(39.1)$ & $33(31.1)$ & \\
\hline Home with services & $3(13)$ & $16(15.1)$ & \\
\hline Hospice & - & $1(0.9)$ & \\
\hline Long-term care & $1(4.4)$ & $2(1.9)$ & \\
\hline Psychiatric hospital & - & $2(1.9)$ & \\
\hline Morgue & $3(13)$ & $8(7.6)$ & \\
\hline Rehab (inpatient) & $5(21.7)$ & $13(12.3)$ & \\
\hline Skilled nursing facility & $2(8.7)$ & $27(25.5)$ & \\
\hline CT within 6 hours of arrival, $n(\%)$ & $5(21.7)$ & $46(43.4)$ & 0.063 \\
\hline Time to CT (minutes) & $134.6 \pm 63.9$ & $143.9 \pm 74.8$ & 0.849 \\
\hline
\end{tabular}

$E D$, emergency department.

other centers. In addition, we compared the cohort of patients exposed to the DA protocol with a contemporaneous control cohort that was not exposed to the DA protocol. Although we found no differences in the primary and secondary outcomes, the relatively lower number of patients in the DA cohort raises the possibility of type II error. Importantly, though the mortality rate was slightly higher in the DA cohort, the cause of mortality in each case was not attributable to any aspect of the DA process. In addition, though there were patients in the DA cohort who underwent operating room procedures, we did not observe any direct-to-operating room transfers. Thus, this aspect of the DA pathway needs to be further assessed for feasibility and safety. Finally, the major difference between the DA and non-DA populations was that the patients in the DA protocol were transferred to our institution during Code Help, a period of severe ED congestion, whereas those in the ED-to-ED pathway were transferred during a less congested period. Though the DA protocol specifically bypasses the ED, it is possible that significant ED congestion affects care on the general care wards, ICUs and operating rooms where these patients received care. As such, this may have impacted our results in a manner that we could not predict.

\section{CONCLUSION}

DA is a feasible method to transfer patients to a high-level trauma center when the ED is overcrowded. Adoption of DA may facilitate patient transfer to level 1 or 2 trauma centers and assist in providing timely care for critically injured patients.
Contributors KCS and PFD participated in the concept, design, data acquisition, analysis, interpretation and drafting of the article. AG and KB participated in the design, data acquisition, analysis, interpretation and drafting of the article. MS participated in the concept, design and drafting of the article. AR participated in the concept, design and critical revisions of the article. GCV participated in the concept, design, data acquisition and drafting of the article.

Funding The authors have not declared a specific grant for this research from any funding agency in the public, commercial or not-for-profit sectors.

Map disclaimer The depiction of boundaries on this map does not imply the expression of any opinion whatsoever on the part of BMJ (or any member of its group) concerning the legal status of any country, territory, jurisdiction or area or of its authorities. This map is provided without any warranty of any kind, either express or implied.

Competing interests None declared.

\section{Patient consent for publication Not required.}

Ethics approval The direct admission protocol was implemented as a part of a hospital quality improvement initiative and was exempt from the institutional review. Use of data from patient electronic medical records was approved by the institutional review board of Partners Healthcare, Boston, Massachusetts (institutional review board protocol 2011P01124).

Provenance and peer review Not commissioned; externally peer reviewed.

Data availability statement No data are available. Data for this study are not available.

Open access This is an open access article distributed in accordance with the Creative Commons Attribution Non Commercial (CC BY-NC 4.0) license, which permits others to distribute, remix, adapt, build upon this work non-commercially, and license their derivative works on different terms, provided the original work is properly cited, appropriate credit is given, any changes made indicated, and the use is non-commercial. See: http://creativecommons.org/licenses/by-nc/4.0/.

\section{REFERENCES}

1 Haas B, Jurkovich GJ, Wang J, Rivara FP, Mackenzie EJ, Nathens AB. Survival advantage in trauma centers: expeditious intervention or experience? J Am Coll Surg 2009;208:28-36.

2 Demetriades D, Martin M, Salim A, Rhee P, Brown C, Chan L. The effect of trauma center designation and trauma volume on outcome in specific severe injuries. Ann Surg 2005;242:206-13.

3 Eastman AB, Mackenzie EJ, Nathens AB. Sustaining a coordinated, regional approach to trauma and emergency care is critical to patient health care needs. Health Aff 2013;32:2091-8

4 Schull MJ, Vermeulen M, Slaughter G, Morrison L, Daly P. Emergency department crowding and thrombolysis delays in acute myocardial infarction. Ann Emerg Med 2004:44:577-85

5 Fee C, Weber EJ, Maak CA, Bacchetti P. Effect of emergency department crowding on time to antibiotics in patients admitted with community-acquired pneumonia. Ann Emerg Med 2007;50:501-9.

6 Reznek MA, Murray E, Youngren MN, Durham NT, Michael SS. Door-to-Imaging time for acute stroke patients is adversely affected by emergency department crowding. Stroke 2017;48:49-54.

7 Morley C, Unwin M, Peterson GM, Stankovich J, Kinsman L. Emergency department crowding: a systematic review of causes, consequences and solutions. PLoS One 2018;13:e0203316.

8 Siegel B. The emergency department: rethinking the safety net for the safety net. Health Aff 2004; Suppl Web Exclusives:W4-146.

9 Commonwealth of Massachusetts. Code Help plans for hospitals. Boston, MA. https:// www.mass.gov/lists/code-help-plans-for-hospitals (7 Jul 2020).

10 Michael SS, Broach JP, Kotkowski KA, Brush DE, Volturo GA, Reznek MA. Code help: can this unique state regulatory intervention improve emergency department crowding? West J Emerg Med 2018;19:501-9.

11 Allwes D, 2015. Update - reducing emergency department patient boarding. Commonwealth of Massachusetts; June 3:

12 Branas CC, MacKenzie EJ, Williams JC, Schwab CW, Teter HM, Flanigan MC, Blatt AJ, ReVelle CS. Access to trauma centers in the United States. JAMA 2005;293:2626-33.

13 Gonzalez RP, Cummings G, Mulekar M, Rodning CB. Increased mortality in rural vehicular trauma: identifying contributing factors through data linkage. J Trauma 2006:61:404-9.

14 Newgard CD, Schmicker RH, Hedges JR, Trickett JP, Davis DP, Bulger EM, Aufderheide TP, Minei JP, Hata JS, Gubler KD, et al. Emergency medical services intervals and survival in trauma: assessment of the "golden hour" in a North American prospective cohort. Ann Emerg Med 2010;55:235-46.

15 Hashmi ZG, Jarman MP, Uribe-Leitz T, Goralnick E, Newgard CD, Salim A, Cornwell E, Haider AH. Access delayed is access denied: relationship between access to trauma center care and pre-hospital death. J Am Coll Surg 2019;228:9-20. 